\title{
SKY-HOOK CONTROL AND KALMAN FILTERING IN NONLINEAR MODEL OF TRACKED VEHICLE SUSPENSION SYSTEM
}

\author{
Andrzej JURKIEWICZ*, Janusz KOWAL*, Kamil ZAJĄC* \\ *Faculty of Mechanical Engineering and Robotics, AGH University of Science and Technology, \\ Al. A. Mickiewicza 30, 30-059 Kraków, Poland \\ jurkand@agh.edu.pl, jkowal@agh.edu.pl, kazajac@agh.edu.pl
}

received 10 October 2016, revised 19 September 2017, accepted 21 September 2017

\begin{abstract}
The essence of the undertaken topic is application of the continuous sky-hook control strategy and the Extended Kalman Filter as the state observer in the $2 \mathrm{~S} 1$ tracked vehicle suspension system. The half-car model of this suspension system consists of seven logarithmic spiral springs and two magnetorheological dampers which has been described by the Bingham model. The applied continuous skyhook control strategy considers nonlinear stiffness characteristic of the logarithmic spiral springs. The control is determined on estimates generated by the Extended Kalman Filter. Improve of ride comfort is verified by comparing simulation results, under the same driving conditions, of controlled and passive vehicle suspension systems.
\end{abstract}

Key words: Continuous Sky-Hook Control Strategy, Extended Kalman Filter, Military Vehicle, Nonlinear Model, Semi-Active Suspension System, Simulation

\section{INTRODUCTION}

Effective vibration damping of the hull of a tracked vehicle improves working conditions of vehicle crew, which affects the growth of conducting fire accuracy (Machoczek and Mężyk, 2015; Rybak et al., 2011). As a result, the success of combat operations can be indirectly dependent on properties of the suspension system. It stimulates the development of modern tracked vehicle systems. Nowadays, suspension structures use conventional passive systems and semi active or even active systems. Semiactive systems provide the ability to generate variable damping force and for this reason these systems are control systems. Their primary task is to reduce an impact of changes in shape of substrate on the chassis.

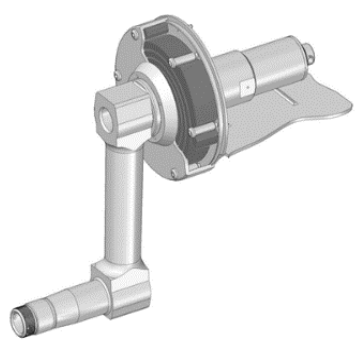

Fig. 1. The logharithmic spiral springs package of the modernized 2S1 tracked vehicle suspension system

Original version of $2 \mathrm{~S} 1$ chassis is equipped with, ia. fourteen road wheels. The suspension system of each road wheels consists a rocker and a torsion bar (Fig. 1), which imparts stiffness in this system (Jurkiewicz et al., 2014; Nabagło et al., 2014). The torsion bar is considered as a relatively simple and reliable solu- tion. The disadvantages of this component are reduction of space in the vehicle and reduction of vehicle resistance against anti-tank mines. In recent years, the project of modernization of the 2S1 suspension system has been performed. The torsion bars have been replaced by logarithmic shaped spiral springs. Damping properties were improved by the compression of oil by the coils of spiral springs. Unfortunately, the impact of control of oil pressure on the vibration transmission characteristics has proved to be insufficient. Based on this observation, the magnetorheological dampers in the theoretical model of 2S1 suspension system have been applied (Nabagło et al., 2015). The chamber of such damper is filled with magnetorheological fluid. This fluid exposed to magnetic field changes its state from free-flowing liquid to semi-solid state so the damping characteristic can be continuously controlled by proper changes of magnetic field (Bajkowski, 2012). One of the advantages resulting from the application of magnetorheological dampers in suspension systems is the possibility of adjusting the damping force to the current driving conditions and to the actual weight of the vehicle, which among others includes the mass of vehicle equipment.

The result of application magnetorheological dampers is semiactive system. Semi-active systems are used to control energy dissipation. For this reason, in this kind of systems it is possible to adopt control algorithms which provide an increase of the ride comfort. In case of military vehicles or special purpose vehicles the ride comfort significantly affects the quality of soldier's work (Jamroziak et al., 2013). The exposure of the human body to the vibrations could cause a muscular, sensory, intellectual and emotional fatigue or even health problems. It follows that the ride comfort is meaningful for the efficiency of operations on the battlefield. The main goal of this work was assumed on this basis. Researches are focused on the structure of a control system which guarantees an increase of the ride comfort. 
In case of ride comfort oriented systems sky-hook control is widely used. Since 1974, the sky-hook control has been mostly used in automobile suspension systems (Lam and Liao, 2003; Simon and Ahmadian, 2001). In recent years, applications of this control in railway vehicles are also considered (Li and Goodall, 1999). Machoczek et. al. applies the sky-hook control theory in model of military multi-wheeled vehicle (2015). Evaluation of suspension is made by comparison of the displacement, velocity and acceleration amplitudes of vehicle hull, measured in the vertical direction. The authors indicate advantages resulting from the usage of semi-active suspension. Moreover, skyhook control algorithm with energy regenerative system has been studied by Sapiński et al. and Snamina et al. (2011, 2009). The interest of sky-hook control strategy is also observed in case of military vehicles. Nabagło et al. notes that sky-hook control strategy may improve ride comfort in tracked vehicles (2015). For purpose of this work, the continuous sky-hook control strategy was applied due to good results observed in so-called quarter-car model of the 2S1 suspension system. Researches have showed reduction of vertical velocity amplitude and increase of ride comfort, in relation to the model of $2 S 1$ with passive suspension system. In this article, the nonlinearity of logarithmic spiral springs was considered in the continuous sky-hook control strategy formulation.

The continuous sky-hook control strategy depends on some state variables of the suspension system, such as the vertical velocity of the sprung mass and the vertical velocities of unsprung masses. The method, which produces the estimates of these unknown variables with high accuracy is the discrete Kalman filter. This algorithm, based on measurements of control signals and variables representing observable behaviour of the system determines the internal state. It is assumed, that in the theoretical model of the 2S1 suspension system the measured output variables are suspension deflections in first and last axles. The socalled half-car model was used in simulation research in this paper.

The application of magnetorheological dampers determine nonlinearity in equation of motion of the tested 2S1 suspension model. For this reason, the Extended Kalman Filter was considered as the appropriate way of state estimation (Julier and Uhlmann, 2004). The Extended Kalman Filter calculates current state on the basis of measurements of control signals and output variables representing observable behaviour of the system. In addition to the observation of a nonlinear system, this method minimizes the impact of disturbances which affect to control process and measurement process. Lindgärde as the main source of those disturbances gives interference that may be related to vibrations generated by the engine of the vehicle (2002). As an alternative measurement of relative velocity of the sprung mass and unsprung mass, Lindgärde uses differentiation of signal generated by suspension deflection sensor which are previously undergone conventional filtration by a low pass Butterworth filter. However, he argues, that the Kalman filter is preferred solution due to smaller estimation error.

The paper is organized as follows: in section II the 2S1 suspension system is described and the theoretical model with magnetorheological dampers is introduced. The continuous sky-hook control strategy which takes into account nonlinearity of logarithmic spiral spring is described in next section. Section IV is devoted to state estimation by the Extended Kalman Filter. In section $\mathrm{V}$ the results of simulation research are presented. Conclusions and observations are included in last section.

\section{THE 2S1 SUSPENSION SYSTEM}

The $2 S 1$ platform is used in many military vehicles of Polish Land Forces among which may be mentioned about self-propelled mortar RAK, self-propelled howitzer M120G, armoured command staff and command vehicles. The original version of $2 \mathrm{~S} 1$ vehicle suspension system uses torsion bars as parts introducing stiffness to the suspension system. Torsion bars have been popular after the Second World War. Stiffness characteristic of these elements is not progressively non-linear. The stiffness does not increase in a non-proportional way, during twisting of the torsion bar.

The modernization of the 2S1 suspension system includes the use of logarithmic spiral springs. Static characteristic of the logarithmic spiral spring is progressive nonlinear, which is shown in Fig. 2.b, is progressive nonlinear for large value of suspension deflection. It should be noted that this characteristic represents the results of laboratory measurements with the use of spring with thickness 3 [mm].

In addition to the progressivity, further advantage of suspension structure based on spiral springs in relation to the original suspension with torsion bars, is improvement of damping properties. This results from the presence of the oil in package of spiral springs. Control of oil pressure is another factor influencing the damping characteristics of the suspension.
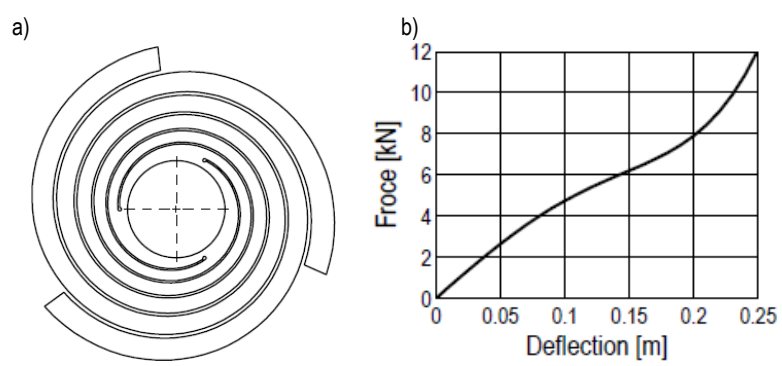

Fig. 2. Logarithmic spiral spring: a) shape of the spring;

b) stiffness characteristic when oil pressure equals 0 [bar]

In order to perform simulation studies the static characteristic of logarithmic spiral spring was described by polynomial approximation of experimental data. It was assumed, that the static characteristic for negative deflection of suspension is a symmetry with respect to the origin. With such assumptions, the approximated function has the following form:

$F_{s}\left(z_{w}\right)=k_{15} z_{w}^{5}+k_{14} z_{w}^{4}+k_{13} z_{w}^{3}+k_{12} z_{w}^{2}+k_{11} z_{w}$

where: $F_{S}$ - approximation of spring force of logarithmic spiral spring, $k_{11} \ldots k_{15}-$ approximation function coefficients, $\mathrm{z}_{w}-$ relative displacement of sprung mass and unsprung mass.

Fig. 1 shows the modified suspension with applied the package of logarithmic spiral springs. The theoretical model of the suspension has been enhanced with magnetorheological damper by Nabagło et al. (2015). Magnetorheological damper is characterized by low power consumption and fast response to changes of current value flowing through the electrical coil. However, this element causes great difficulties during designing process of control system due to the nonlinear dynamic (Spencer et al., 1997). Two magnetorheological dampers generate force which can be determined by the following Bingham model (Sapiński and Filuś, 2003): 


$$
\begin{aligned}
& F_{t 1}=\frac{2}{\pi}\left(f_{a}+f_{b} i_{1}\right) \operatorname{arctg}\left(p v_{11}\right)+v_{11}\left(c_{a}+c_{b} i_{1}\right), \\
& F_{t 7}=\frac{2}{\pi}\left(f_{a}+f_{b} i_{7}\right) \operatorname{arctg}\left(p v_{17}\right)+v_{17}\left(c_{a}+c_{b} i_{7}\right) .
\end{aligned}
$$

In above equations, $v_{11}$ and $v_{17}$ are relative velocities between the damper pistons and the damper rods respectively. Quantities $i_{1}$ and $i_{7}$ are the electric current intensity signals. Elements $f_{a}+f_{b} i_{1}$ and $f_{a}+f_{b} i_{7}$ are Coulomb friction coefficients. Elements $c_{a}+c_{b} i_{1}$ and $c_{a}+c_{b} i_{7}$ describe the viscous friction coefficients.

Before formulating the theoretical model, following assumptions have been made. It was assumed that temperature does not affect magnetorheological fluid properties, as well as lack of leakage of this fluid. The nonlinear stiffness characteristic associated with spiral springs has been adopted. Linear stiffness and linear damping of road wheels were assumed. The sprung mass and unsprung masses were concentrated in points. Furthermore, bilateral constraints were assumed. It means that road wheels always have a contact with substrate. The theoretical model for such simplified structure of the $2 S 1$ tracked vehicle suspension system (Fig. 2) is shown below. Equation of motion of unsprung mass in first axle has following form:

$m_{1} \ddot{z}_{1}=F_{k 21}+F_{c 21}-F_{k 11}-F_{t 1}$.

For unsprung masses in axles from the second to the sixth these equations are as follows (index $i$-axle number):

$m_{i} \ddot{z}_{i}=F_{k 2 i}+F_{c 2 i}-F_{k 1 i}$.

Equation for unsprung mass in the last axle:

$m_{7} \ddot{z}_{7}=F_{k 27}+F_{c 27}-F_{k 17}-F_{t 7}$.

Dynamics of vertical motion of the centre of gravity of the vehicle hull has been described in a manner as shown below:

$m \ddot{z}=F_{t 1}+F_{t 1}+\sum_{j=1}^{7} F_{k 1 j}$.

Dynamic equation of rotational motion of the vehicle hull around the pitch axis has been described by the following equations:

$J_{x} \ddot{\varphi}_{x}=\sum_{j=5}^{7} l_{j} F_{k 1 j}-\sum_{j=1}^{4} l_{j} F_{k 1 j}+l_{7} F_{t 7}-l_{1} F_{t 1}$.

Constants $k_{21} \ldots k_{27}$ and $c_{21} \ldots c_{27}$ are stiffness and damping coefficients of respective road wheels. $J_{x}$ defines moment of inertia around the pitch axis. Constants $l_{1} \ldots l_{7}$ expresses distance from the centre of gravity of the vehicle hull to further axles. Finally, quantity $\mathrm{d}$ is half of the vehicle hull width. Signals $g_{1} \ldots g_{7}$ represent external excitation signals as road profile functions travelled by the vehicle road wheels. It can be therefore concluded that control of suspension system is an example of vibration isolation system. Variables $F_{k 1} \ldots F_{k 7}$ are forces exerted by compressed logarithmic spiral springs being part of the 2S1 suspension system. According to (1) these forces can be described as follows:

$F_{k 1 i}=F_{s}\left(z_{w i}\right)$

where index $i$ means number of vehicle axle so $z_{w i}$ are relative displacements of sprung mass and unsprung masses. These variables, for first four axles, can be calculated according to the following equation:

$z_{w i}=z-z_{i}+l_{i} z_{i}$

In next three axles of vehicle, the relative displacements of sprung mass and unsprung masses are described by:

$$
z_{w i}=z-z_{i}-l_{i} z_{i} \text {. }
$$

Prior to the formulation of state-space model based on equations (4) - (16) following state variables were assumed: $x_{1} \ldots x_{14}-$ displacements and velocities of the unsprung masses; $x_{15}$ and $x_{16}$ - displacement and velocity of the sprung mass; $x_{17}$ and $x_{18}$ - angles and angular velocities around pitch axis. The electric current intensity signals $i_{1}$ and $i_{7}$ were described by control $u_{1}$ and $u_{2}$. The substrate profiles and derivatives of the road profile signals were assumed as control $u_{3} \ldots u_{16}$. The state-space representation of the simplified 2S1 suspension system (Fig. 3) for such variables has form:

$$
\left[\begin{array}{c}
\dot{x}_{1} \\
\dot{x}_{2} \\
\dot{x}_{3} \\
\dot{x}_{4} \\
\dot{x}_{5} \\
\dot{x}_{6} \\
\dot{x}_{7} \\
\dot{x}_{8} \\
\dot{x}_{9} \\
\dot{x}_{10} \\
\dot{x}_{11} \\
\dot{x}_{12} \\
\dot{x}_{13} \\
\dot{x}_{14} \\
\dot{x}_{15} \\
\dot{x}_{16} \\
\dot{x}_{17} \\
\dot{x}_{18}
\end{array}\right]=\left[\begin{array}{c}
x_{2} \\
\frac{1}{m_{1}}\left(F_{k 21}+F_{c 21}-F_{k 11}-F_{t 1}\right) \\
\frac{1}{m_{1}}\left(F_{k 21}+F_{c 21}-F_{k 11}-F_{t 1}\right) \\
x_{6} \\
\frac{1}{m_{1}}\left(F_{k 21}+F_{c 21}-F_{k 11}-F_{t 1}\right) \\
x_{8} \\
\frac{1}{m_{1}}\left(F_{k 21}+F_{c 21}-F_{k 11}-F_{t 1}\right) \\
x_{10} \\
\frac{1}{m_{1}}\left(F_{k 21}+F_{c 21}-F_{k 11}-F_{t 1}\right) \\
x_{12} \\
\frac{1}{m_{1}}\left(F_{k 21}+F_{c 21}-F_{k 11}-F_{t 1}\right) \\
x_{14} \\
\frac{1}{m_{1}}\left(F_{k 21}+F_{c 21}-F_{k 11}-F_{t 1}\right) \\
x_{16} \\
m^{-1}\left(F_{t 1}+F_{t 1}+\sum_{j=1}^{7} F_{k 1 j}\right) \\
x_{18} \\
\frac{1}{J_{x}}\left(\sum_{j=5}^{7} l_{j} F_{k 1 j}-\sum_{j=1}^{4} l_{j} F_{k 1 j}+l_{7} F_{t 7}-l_{1} F_{t 1}\right)
\end{array}\right]
$$

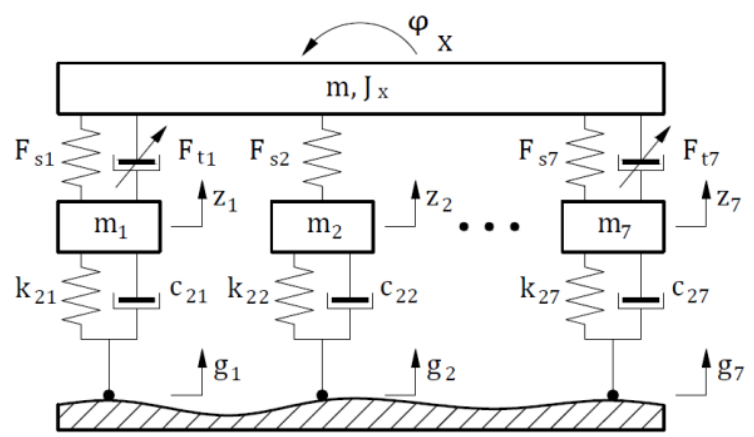

Fig. 3. Simplified model of the $2 \mathrm{~S} 1$ suspension system where: $F_{t 1}, F_{t 7}$ - forces generated by magnetorheological dampers; $F_{s 1} \ldots F_{S 7}$ - forces generated by logarithmic spiral springs; $k_{21} \ldots k_{27}$ and $c_{21} \ldots c_{27}$ - stiffness and damping coefficients of road wheels; $m, J_{x}$ - sprung mass and its moment of inertia; $\varphi_{x}$ - angle of vehicle hull's rotational motion around pitch axis; $z_{1} \ldots z_{7}$ - vertical displacements of unsprung masses; $g_{1} \ldots g_{7}-$ external excitation signals of road profile functions

Above formula describes nonlinear multidimensional statespace system. Suspension deflections in axes of magnetorheological dampers were set as the measured output signals. The output 
matrix was adopted as:

$$
\left.\begin{array}{rrrrrrrrr} 
& H= \\
\text { Col. } & 1 & & 13 & & 15 & & 17 & 18 \\
0 & \cdots & 1 & \cdots & -1 & \cdots & -l_{7} & 0
\end{array}\right] .
$$

Finally, the output equation has the form:

$y=H x$

where: $y$ is 2-dimensional vector of observable state variables and $x$ is 18-dimensional state vector.

\section{THE SKY-HOOK CONTROL STRATEGY}

The sky-hook control has been proposed by Karnopp et al. (1974). In ideal case a viscous damper is connected with sprung mass and abstract sky which is fixed in stationary coordinate system. Such configuration reduces the vertical vibration of the sprung mass, which directly translates into increase of ride comfort. However, this configuration is not physically realizable. Application of an element, between sprung and unsprung masses, with controllable damping characteristic is a method to realize skyhook control strategy. For purposes of this article the continuous sky-hook control strategy was applied in half-car model of the 2S1 tracked vehicle suspension system. The force generated by abstract viscous damper connecting the centre of gravity of the vehicle hull with inertial frame is defined as follows (Machoczek and Mężyk, 2015):

$F_{s k y}=\zeta_{s k y} b_{c r} \dot{z}$.

Parameter $\zeta_{s k y}$ is a non-dimensionless quantity which in the case of critical damping is equal 1 . Critical damping coefficient $b_{c r}$ for the adopted model of the $2 \mathrm{~S} 1$ suspension system has the following form:

$b_{c r}=2 \sqrt{m \boldsymbol{K}}$

where: $\boldsymbol{K}[\mathrm{N} / \mathrm{m}]$ is stiffness of logarithmic spiral springs. This parameter is specified in more details at the end of this chapter. Substitution of (16) to (15) gives force which abstract viscous damper should generate. To adopt the sky-hook control strategy, this force must be equal to the force produced by two magnetorheological dampers

$\mathrm{F}_{t 1}+\mathrm{F}_{t 2}=2 \zeta_{s k y} \dot{Z} \sqrt{m K}$.

Because vibration control of the $2 \mathrm{~S} 1$ suspension system is investigated only in vertical direction it was assumed that $\mathrm{F}_{t 1}=$ $\mathrm{F}_{t 7}$. Hence, these forces are equal:

$\mathrm{F}_{t 1}=\mathrm{F}_{t 2}=\zeta_{s k y} \dot{z} \sqrt{m \boldsymbol{K}}$.

This equation can be considered as a control law. To apply this law, it is necessary to introduce inverse models of magnetorheological dampers. The inverse of equations (2) - (3) due to variables $i_{1}$ and $i_{7}$ leads to:

$i=\frac{\zeta_{s k y} \dot{z} \sqrt{m K}-2 \pi^{-1} f_{a} \operatorname{arctg}(p v)-c_{a} v}{2 \pi^{-1} f_{b} \operatorname{arctg}(p v)+c_{b} v}$.

Appropriate substitution of variables $v_{1}$ and $v_{7}$ to $v$ leads to current intensity signals $i_{1}$ and $i_{7}$. The flow of electrical currents $i_{1}$ and $i_{7}$ through the windings of magnetorheological dampers should obtain the desired values of damping forces $\mathrm{F}_{t 1}$ and $\mathrm{F}_{t 7}$. Variables $i_{1}$ and $i_{7}$ are the input variables of the state space model (12) designated as $u_{1}$ and $u_{7}$. Vertical velocity $z$ of the sprung mass is state variable $x_{16}$. Variables $v_{1}$ and $v_{7}$ are relative velocities between the unsprung masses $m_{1}, m_{7}$ and sprung mass $m$. Therefore, following equations can be written:

$v_{1}=x_{2}-x_{16}+l_{1} x_{18}$

$v_{7}=x_{14}-x_{16}-l_{7} x_{18}$.

Since the stiffness characteristic of logarithmic spiral springs (1) is non-proportional to the suspension deflection, the stiffness parameter $\boldsymbol{K}$ is computed as a sum of proper slopes of tangent lines to the curve presented in Fig. 2.b. Modelled suspension system consists of seven springs with nonlinear stiffness characteristics so $\boldsymbol{K}$ could be described as:

$\boldsymbol{K}=\sum_{i=1}^{7} k_{1 i}$.

Each $k_{1 i}$ where $i=1 \ldots 7$ are slopes of tangent lines to the function (1) calculated in current value of deflections:

$k_{1 i}=\left[\frac{\partial F_{S}\left(z_{w}\right)}{\partial z_{w}}\right]_{z_{w i}}$.

Substitution of the proper values of variables $z_{w i}$, which are showed in (10) and (11), leads to the stiffness parameter $\boldsymbol{K}$ :

$\boldsymbol{K}=\sum_{i=1}^{7}\left[\frac{\partial F_{S}\left(z_{w}\right)}{\partial z_{w}}\right]_{z_{w i}}$.

Finally, the inverse model of magnetorheological damper has the form:

$i=\frac{\zeta_{s k y} \dot{z} \sqrt{m \sum_{i=1}^{7}\left[\frac{\partial F_{S}\left(z_{w}\right)}{\partial z_{w}}\right]_{z_{w i}}}-2 \pi^{-1} f_{a} \operatorname{arctg}(p v)-c_{a} v}{2 \pi^{-1} f_{b} \operatorname{arctg}(p v)+c_{b} v}$.

Above equation is used to compute electric current intensity signals which control the magnetorheological dampers.

\section{THE EXTENDED KALMAN FILTER}

R. E. Kalman has presented modern idea of state estimation in 1960 at National Aeronautics and Space Administration (NASA) Ames Research Centre in Mountain View, California. The method of R. E. Kalman was considered as a possible solution to the problem of estimating trajectories of spacecraft which were intended to be used during manned flights to the Moon. In 1961, the research team led by Stanley F. Schmidt has conducted simulation study of navigation system taking into consideration the Kalman's method. Stanley F. Schmidt has introduced modifications in the standard algorithm to obtain possibly the most accurate approximations of the state vector in nonlinear system. The result of his work is the Extended Kalman Filter which is now the most widely used method of state estimation in nonlinear systems.

The Kalman Filter is a minimum mean-squared error estimator of the sate vector (Becerra et al., 2001; Julier and Uhlmann, 2004). It is a result of minimizing expected value of quadratic cost function $J=E\left[\left(x-\hat{x}_{k}\right)\left(x-\hat{x}_{k}\right)^{T}\right]$, where $x \in \mathcal{R}^{n}$ is a state vector and $\hat{x}_{k} \in \mathcal{R}^{n}$ is an estimator of this vector. Minimization of the cost function $J$ leads to:

$\hat{x}_{k}=E\left[x \mid Y_{k}\right]$.

$Y_{k}$ is a vector which has the form $\left[\mathrm{y}\left(\mathrm{t}_{1}\right), \mathrm{y}\left(\mathrm{t}_{2}\right), \ldots, \mathrm{y}\left(\mathrm{t}_{k}\right)\right]$, where $\mathrm{y}\left(\mathrm{t}_{i}\right) \in \mathcal{R}^{p}$ for all $i \in\{1 \ldots k\}$ are vectors of discrete 
system output. Above formula determines the direction of meansquared estimation, which considers events related to the estimated parameter.

The process control and observed measurements in the 2S1 suspension system may be distributed due to noises. In the following, it is assumed that the process noise $w(t)$ is additive noise. The random excitations signals adopted as the additive process noise may be caused by air resistance or friction in contact between suspension elements. Their source may also be engine or other machinery of the vehicle equipment. The output vector $y(t)$ is measured by a sensor which is corrupted by an additive measurement noise $v(t)$ also. To apply standard algorithm of the Extended Kalman Filter, the random signals $w(t)$ and $v(t)$ are assumed as stationary random processes. Positivedefinite, constant in time, the diagonal matrix $Q \in \mathcal{R}^{n \times n}$ is the covariance matrix of the process noise $w(t) \in \mathcal{R}^{n}$. Matrix $R \in \mathcal{R}^{p \times p}$ is a covariance matrix of measurement noise $v(t) \in$ $\mathcal{R}^{p}$ and is also positive definite, constant in time and diagonal. The expected values $E[w(t)]$ and $E[v(t)]$ are equal to zero. Moreover, random vectors $w(t)$ and $v(t)$ are assumed as Gaussian white noise. Considering mentioned disturbances, the model described by equations (12) and (14) now has the following form (Becerra et al., 2001):

$\dot{x}(t)=f(x(t), u(t))+w(t)$,

$y(t)=h(x(t))+v(t)$.

And finally, it is assumed that the expected value $E\left[w(t) v(t)^{T}\right]$ is equal to zero matrix, which means that the process noise and measurement noise are not correlated.

Implementation of the Extended Kalman Filter in a digital control unit requires a discrete form of equations (27) and (28). These equations, using the definition of a derivative, assuming a step size $\Delta$ and $t=t_{k}$, take the from:

$x\left(t_{k+1}\right)=f\left(x\left(t_{k}\right), u\left(t_{k}\right)\right) \Delta+w\left(t_{k}\right) \Delta+x\left(t_{k}\right)$,

$x\left(t_{k+1}\right)=h\left(x\left(t_{k+1}\right), u\left(t_{k}\right)\right)+v\left(t_{k+1}\right)$.

The model (29) describes the so-called discrete-time GaussMarkov random process. It follows from the fact, that $w(t)$ is additive Gaussian random vector. Whereas, the Markov property stems from the fact that system state at time $t_{k}$ depends only on the state at the previous time $t_{k-1}$.

The Extended Kalman Filter is two-phase state estimation algorithm of non-linear dynamic system. The idea of this method is to linearize the non-linear system at a point in state-space whose coordinates specify current value of the estimator and to apply the linearized description to calculate next estimate (Julier and Uhlmann, 2004). The Extended Kalman Filter is first order approximation of the standard Kalman filter because the linearization is based on first order Taylor series expansion. Estimation process, which is determined by (26), has following structure:

$$
\begin{aligned}
& \hat{x}\left(t_{k} \mid t_{k-1}\right)=f\left(\hat{x}\left(t_{k-1} \mid t_{k-1}\right), u\left(t_{k-1}\right)\right) \Delta+\hat{x}\left(t_{k-1} \mid t_{k-1}\right), \\
& P_{k \mid k-1}=A_{k-1} P_{k-1 \mid k-1} A_{k-1}^{T}+Q, \\
& K_{k}=P_{k \mid k-1} H_{k}^{T}\left[H_{k} P_{k \mid k-1} H_{k}^{T}+R\right]^{-1}, \\
& \hat{x}\left(t_{k} \mid t_{k}\right)=\hat{x}\left(t_{k} \mid t_{k-1}\right)+K_{k}\left(y\left(t_{k}\right)-h\left(\hat{x}\left(t_{k} \mid t_{k-1}\right)\right)\right), \\
& P_{k \mid k}=\left[I-K_{k} H_{k}\right] P_{k \mid k-1}\left[I-K_{k} H_{k}\right]^{T}+K_{k} R K_{k}^{T}
\end{aligned}
$$

where: $\hat{x}\left(t_{k} \mid t_{k-1}\right)$ and $\hat{x}\left(t_{k} \mid t_{k}\right)$ are a priori and a posteriori estimators of the state vector and they are associated with prediction and update phase respectively. $P_{k \mid k-1}$ and $P_{k \mid k}$ are error covariance matrices of these estimators. The matrix $K_{k}$ is called the Kalman gain. $A_{k-1}$ is the matrix of partial derivatives of nonlinear function (27) with respect to vector $x$. For discrete system, it has form:

$A_{k-1}=\left[I+\Delta\left[\frac{\partial f}{\partial x}\right]_{\left(\hat{x}\left(t_{k-1} \mid t_{k-1}\right), u\left(t_{k-1}\right)\right)}\right]$.

Finally, $H_{k} \in \mathcal{R}^{p \times n}$ is the matrix of partial derivatives of function (28) with respect to vector $x$. This matrix does not change its form since the output equation is linear:

$H_{k}=H$.

\section{SIMULATION RESEARCH}

In simulation research, which was carried out in MATLAB, following assumptions have been adopted. It was assumed, that the $2 \mathrm{~S} 1$ tracked vehicle enters on elevation of height $0.1[\mathrm{~m}]$ with velocity $0.832[\mathrm{~m} / \mathrm{s}]$. In the first stage of study, the passive suspension was examined. In this case, values of electrical currents $i_{1}$ and $i_{7}$ flowing through the windings of magnetorheological dampers were set to $0[A]$. Values of damping forces of each magnetorheological damper result only from the relative displacements $v_{1}$ and $v_{7}$. In the next stage of study, the continuous sky-hook control strategy was tested. The control strategy is defined by equation (25).

Furthermore, the presence of disturbances affecting control process and measurement process was introduced. These disturbances were set as white noise. The Extend Kalman Filter was introduced to minimize their impact as the state estimator in passive case and in control case.

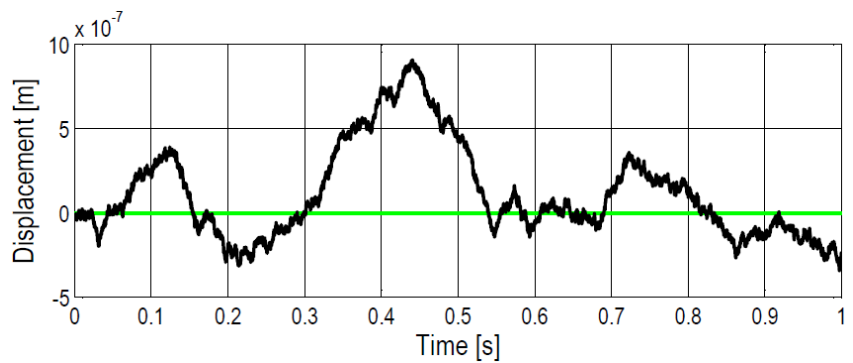

Fig. 4. Estimation of vertical displacement of the centre of the gravity of the vehicle hull and response of the system not affected by white noise

Fig. 4 shows the vertical displacement of the centre of gravity of the $2 S 1$ tracked vehicle in which semi-active suspension is controlled by the continuous sky-hook control strategy. The graph shows the influence of the Extended Kalman Filter in the system affected by process noise and measurement noise. The graph shows also the response of the system in which process noise and measurement noise have not been introduced and the algorithm of Extended Kalman filter has not been used. Initialization of the algorithm requires assumptions about the initial value of a posteriori estimator and the initial value of a posteriori error covariance matrix. The estimator of the state is a random vector whose values are depended on the error covariance matrix. In- 
crease of accuracy estimation results from update this matrix in each discrete step time.

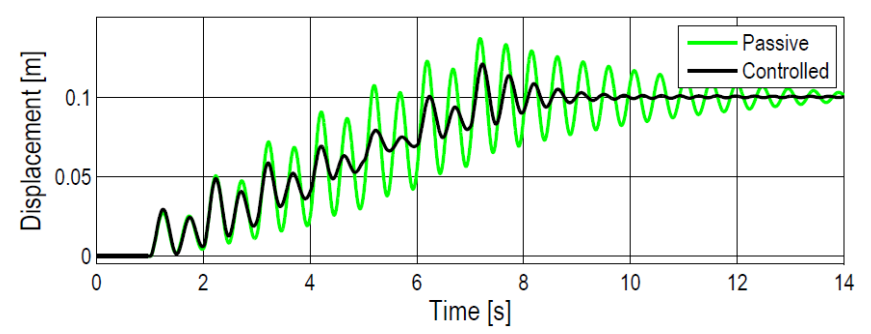

Fig. 5. Vertical displacement of the centre of the gravity of the vehicle hull in passive and controlled systems

The above Fig. 5 shows vertical displacement of the centre of the vehicle hull in passive suspension system and controlled suspension system. Fig. 6 is a comparison of the vertical velocity of the centre of the vehicle hull between passive suspension system and controlled suspension system, in which formulated continuous sky-hook control strategy was applied. Time-domain analysis show the advantage of proposed semi-active suspension system.

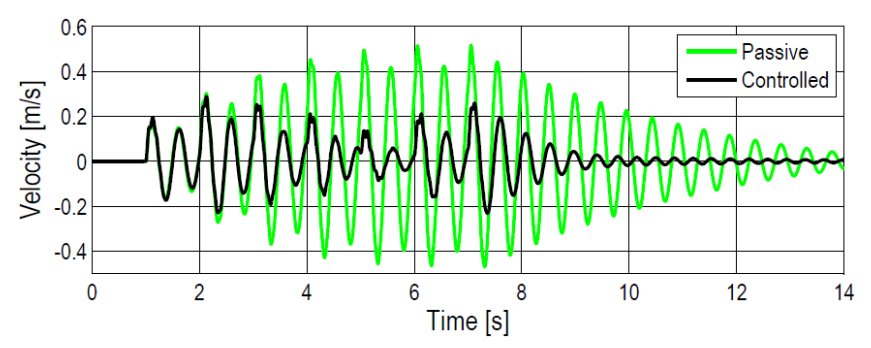

Fig. 6. Vertical velocity of the centre of the gravity of the vehicle hull in passive and controlled systems

In the following stage of the simulation research, two vibration transmissibility characteristics were made. The transmissibility characteristic is the key measure of quality of the vibration control system. Fig. 7 shows logarithmic transmissibility characteristics from the input $u$ to the vertical displacement of the centre of the gravity of the vehicle hull, which is designed as $x_{17}$. It should be mentioned that the current values flowing through the windings of magnetorheological dampers can vary in range from 0 to $5[A]$.

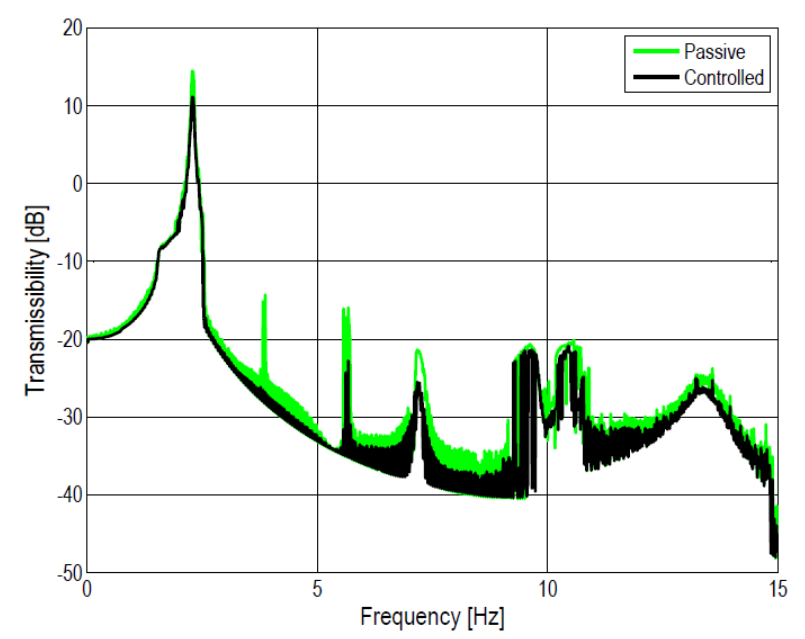

Fig. 7. Logarithmic vibration transmissibility characteristics of the tracked vehicle hull
To compare transmissibility function values in selected frequencies the following characteristic are introduced. The graph shows the result of dividing the value of the dimensionless transmissibility characteristic of passive system by the value of the dimensionless transmissibility characteristic of controlled system, which is finally multiplied by $100[\%]$.

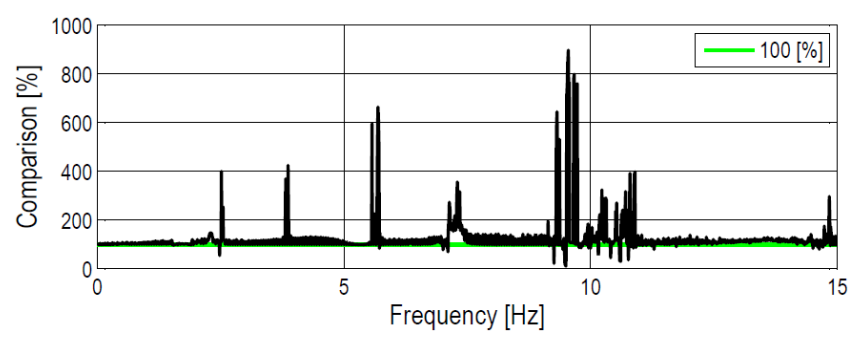

Fig. 8. Comparison of dimensionless transmissibility characteristic values in passive and controlled suspension system

\section{CONCLUSION}

Estimated state variables provide the possibility to control the 2S1 suspension model through the continuous sky-hook control strategy. On the basis on measurements of substrate profile and two physical variables, which are relative displacements between the damper pistons and the damper rods, eighteen state variables are estimated. The use of the Extended Kalman Filter brings many simplifications. The Extended Kalman Filter does not bring delays which occur in the case of conventional filtration where filters like Butterworth filter, Chebyshev filter etc. are applied. The problem may be implementation of this algorithm in digital control unit.

Time characteristics (Fig. 5, 6) indicate that the continuous sky-hook control strategy takes into account the nonlinearity of spiral springs reduces amplitudes of vertical displacement and vertical velocity of the centre of gravity of the vehicle hull and shortens the time needed to achieve the appropriate level. Comparison of vertical velocities of the vehicle with controlled suspension and with passive suspension leads to achieve the increase of ride comfort. Also, this can have a positive affect the function of gun stabilization system which means the increase in accuracy of conducting fire.

Vibration transmissibility characteristics, which are presented in Fig. 7, show that the continuous sky-hook control strategy is effective. The transmissibility characteristics in natural frequency of the vehicle hull have significantly lower values in case when the continuous sky-hook control strategy is applied. Also, it should be mentioned, that the proposed control strategy, in compare to passive suspension system leads to lower values of displacement amplitudes in range between natural frequencies of the sprung mass and unsprung masses. This fact is confirmed by the Fig. 8 . It can be assumed, that there is an increase of ride comfort of the vehicle crew in whole range of frequency of excitation signal. In case of linear suspension model similar result was achieved by Laalej et al. (2012). During work over formulating sky-hook control strategy, the nonlinear characteristic of spiral spring has been considered in this article. 


\section{REFERENCES}

1. Bajkowski J. M. (2012), Design, Analysis and Performance Evaluation of the Linear, Magnetorheological Damper, Acta Mechanica et Automatica, 6, 5-9.

2. Becerra V. M., Roberts P. D., Griffiths G. W. (2001), Applying the extended Kalman filter to systems described by nonlinear differentialalgebraic equations, Control Engineering Practice, 9(3), 267-281.

3. Jamroziak K., Kosobudzki M., Ptak J. (2013), Assessment of the comfort of passenger transport in special purpose vehicles, Eksploatacja i Niezawodność, 15(1), 25-30.

4. Julier S.J., Uhlmann J.K. (2004), Unscented filtering and nonlinear estimation, Proceedings of the IEEE, 92(3), 401-422.

5. Jurkiewicz A., Nabagło T., Kowal J., Apostoł M. (2014), A new suspension system of an autonomous caterpillar platform, Journal of Theoretical and Applied Mechanics, 52(4), 857-867.

6. Karnopp D., Crosby M. J., Harwood R. A. (1974), Vibration control using semi-active force generators, Journal of Engineering for Industry, 96(2), 619-626.

7. Laalej H., Lang Z. Q., Sapinski B., Martynowicz P. (2012), MR damper based implementation of nonlinear damping for a pitch plane suspension system, Smart Materials and Structures, 21(4), 045006.

8. Lam A. H. F., Liao W. H. (2003), Semi-active control of automotive suspension systems with magneto-rheological dampers, International Journal of Vehicle Design, 33(1-3), 50-75.

9. Li H., Goodall R. M. (1999), Linear and non-linear skyhook damping control laws for active railway suspensions, Control Engineering Practice, 7(7), 843-850.

10. Lindgärde 0. (2002). Kalman filtering in semi-active suspension control, Proceedings of 15th IFAC World Congress,1539-1544.

11. Machoczek T., Mężyk A. (2015), The multi-wheeled vehicle's suspension control (in Polish), Przegląd Mechaniczny, 1, 19-25.
12. Nabagło T., Jurkiewicz A., Apostoł M., Micek P. (2014), Simulation of $2 \mathrm{~S} 1$ tracked vehicle model with modernized suspension system during crossing a single obstacle, Solid State Phenomena, Trans Tech Publications, 208, 140-147.

13. Nabagło T., Jurkiewicz A., Kowal J. (2015), Semi-active suspension system for $2 \mathrm{~S} 1$ tracked platform in application of improvement of the vehicle body stability, Applied Mechanics and Materials, 759, 77-90.

14. Rybak P., Borkowski W., Wysocki J., Hryciów Z., Michałowski B. (2011), Model research of the light tank basing on multi-task combat platform (in Polish), Szybkobieżne Pojazdy Gąsienicowe, 2, 39-46.

15. Sapiński B., Filuś J. (2003), Analysis of parametric models of MR linear damper, Journal of Theoretical and Applied Mechanics, 41(2), 215-240.

16. Sapiński B., Jastrzębski Ł., Węgrzynowski M. (2011), Modelling of a self-powered vibration reduction system (in Polish), Modelowanie Inżynierskie, 10(41), 353-362.

17. Simon D., Ahmadian M. (2001), Vehicle evaluation of the performance of magneto rheological dampers for heavy truck suspensions, Journal of Vibration and Acoustics, Transactions of the ASME, 123(3), 365-375.

18. Snamina J., Podsiadło A., Orkisz P. (2009), Skyhook vibration control with energy regenerative system, Mechanics, 28, 124-130.

19. Spencer Jr. B. F., Dyke S. J., Sain M. K., Carlson J. (1997), Phenomenological model for magnetorheological dampers, Journal of engineering mechanics, 123(3), 230-238.

The work has been accomplished under the research project No. 15.11.130.429 financed by the grant of Faculty of Mechanical Engineering and Robotics 